\section{Foetal haemoglobin, blood transfusion, and retinopathy of prematurity in very preterm infants: a pilot prospective cohort study}

CJ Stutchfield ${ }^{1,2}$, A Jain 1, D Odd ${ }^{2}$, C Williams 3,4 and R Markham ${ }^{3}$

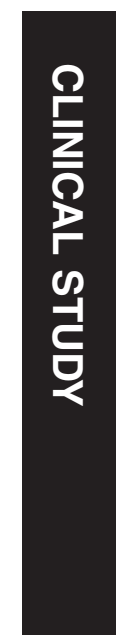

\begin{abstract}
Purpose To identify if there is an association between foetal haemoglobin ( $\mathrm{HbF}$ ) concentration and retinopathy of prematurity (ROP) in very preterm infants.

Patients and methods Prospective cohort study. Infants born $<32$ weeks' gestational age or $<1501 \mathrm{~g}$ in two tertiary neonatal units between January 2012 and May $2013(n=42)$ were enrolled. $\mathrm{HbF}$ and adult haemoglobin (HbA) concentrations were measured using high-pressure liquid chromatography from blood samples sent as part of routine neonatal care once routinely requested laboratory tests had been performed. Clinical data were obtained from case notes. We calculated odds ratios (ORs) $(95 \%$ confidence intervals (CIs)) to quantify the relationship between initial and mean $\% \mathrm{HbF}$ with ROP severity (none, stages 1-3).

Results A total of 42 infants were recruited: mean gestation 28.0 weeks (SD 1.91); mean birth weight $1042 \mathrm{~g}$ (SD 264). Six infants died before ROP screening; 14/36 developed ROP (39\%); and 22/36 (61\%) did not. Infants who developed ROP had similar initial $\% \mathrm{HbF}$

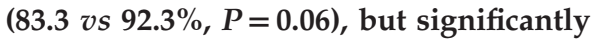
lower mean \% HbF (61.75 vs $91.9 \%$, $P=0.0001)$ during their inpatient stay than those who did not develop ROP. In ordinal logistic regression models adjusted for birth weight, gestation and transfusion volume, mean post-natal $\% \mathrm{HbF}$ was negatively associated with ROP severity: adjusted OR $0.94(0.90-0.99)$, while initial $\% \mathrm{HbF}$ at birth was not: adjusted OR 1.05 (0.97-1.16). Conclusion Replacing $\mathrm{HbF}$ by $\mathrm{HbA}$ during transfusion may promote ROP development by rapidly increasing oxygen availability to the
\end{abstract}

retina. Conversely, maintaining a higher $\% \mathrm{HbF}$ may be a protective factor against ROP. Eye (2017) 31, 1451-1455; doi:10.1038/eye.2017.76; published online 26 May 2017

Introduction

Studies in preterm neonates have highlighted that a balance must be found between the toxic effects of high-oxygen saturations, and the increased morbidity and mortality associated with targeting lower saturations. ${ }^{1-4}$ Birth weight and gestation have also been shown to be consistently independently associated with ROP development. A number of other factors appear to have an association with ROP, including genetics, poor nutrition and poor weight gain, sepsis, and necrotising enterocolitis. ${ }^{5-7}$ Various studies have identified an association between ROP and blood transfusion. ${ }^{8-10}$

Neonates have a predominance of foetal haemoglobin $(\mathrm{HbF})$ at birth. Approximately 85\% of total haemoglobin is $\mathrm{HbF}$ in infants born at 35 weeks' gestation, which gradually declines until it has disappeared by the age of 1-2 years. ${ }^{11,12} \mathrm{HbF}$ has a greater affinity for oxygen compared to $\mathrm{HbA}$, shifting the haemoglobinoxygen dissociation curve to the left, causing preferential foetal oxygen binding in utero. However, $\mathrm{HbF}$ preponderance postnatally in very preterm infants leads to greater difficulty unloading oxygen to the tissues. This is partly offset by a steeper oxygen-haemoglobin dissociation curve than that for $\mathrm{HbA}$, but exacerbated as the levels of 2,3diphosphoglycerate (a product of glycolysis that promotes oxygen release from oxyhaemoglobin) are low in preterm neonates. ${ }^{13}$
${ }^{1}$ Neonatal Intensive Care Unit, St Michael's Hospital, Bristol, UK

${ }^{2}$ Neonatal Intensive Care Unit, Southmead Hospital, Bristol, UK

${ }^{3}$ Bristol Eye Hospital, University Hospitals Bristol NHS Foundation Trust, Bristol, UK

${ }^{4}$ School of Social and Community Medicine, University of Bristol, Bristol, UK

Correspondence:

CJ Stutchfield, Paediatric Intensive Care Unit, Bristol Children's Hospital, Paul O'Gorman Building, Upper Maudlin St, Bristol, BS2 8BJ, UK

Tel: +44 (0)11 7923 0000; Fax: +44 (0)11 73428538 . E-mail: cstutch@gmail.com

Received: 15 March 2016 Accepted in revised form: 6 April 2017 Published online: 26 May 2017 
Anaemia of prematurity, in part thought to be related to reduced red cell life span and low erythropoietin levels, is exacerbated by clinical blood sampling, leading to the need for blood transfusion in many very preterm infants. ${ }^{14}$ De Halleux et al ${ }^{15}$ demonstrated clinically that the oxygen-haemoglobin dissociation curve is shifted to the right in preterm infants after blood transfusion. We hypothesised that as the $\mathrm{HbF}: \mathrm{HbA}$ ratio decreases with blood transfusion, more oxygen is made available to the developing retina for any given arterial partial pressure of oxygen $\left(\mathrm{PaO}_{2}\right)$, possibly contributing to ROP

development by increasing the oxygen availability in the developing retina and reducing angiogenic drive. We aimed to explore whether there might be an association between either initial \% $\mathrm{HbF}$ (on admission after birth) and/or the mean inpatient $\mathrm{HbF} \%$ (during their hospital admission), with the development of ROP in very preterm infants.

\section{Materials and methods}

\section{Study design}

We conducted a pilot prospective cohort study across two tertiary neonatal intensive care units in Bristol, UK (St Michael's and Southmead Hospital). All inborn infants and those retrieved from neighbouring hospitals within $24 \mathrm{~h}$ of birth born $<32$ weeks' gestation or $<1501 \mathrm{~g}$ were eligible for inclusion. Parents were counselled as to the nature of the study and provided with an information leaflet. With informed parental consent, all routine EDTA samples taken during the baby's admission were analysed for $\mathrm{HbF} \%, \mathrm{HbA} \%$, and $\mathrm{HbF}$ : A ratio. When $\mathrm{Hb}$ is mentioned, it is defined whether $\mathrm{HbF}$ or $\mathrm{HbA}$ is being referred to. No additional blood samples were taken for the purposes of the study. Analysis of $\mathrm{HbF}$ and $\mathrm{HbA}$ were performed at a single site (Southmead Hospital) using high-performance liquid chromatography (Bio-Rad, Berkley, CA, USA, Variant II HPLC analyser; daily calibration; $5 \mu$ l blood sample volume). Recruited patients received routine neonatal care and the existing local protocol was followed for blood transfusion thresholds. Infants received on-site retinopathy screening by a single consultant ophthalmologist according to national guidelines. ${ }^{16}$

\section{Data collection}

Patient case notes were reviewed after discharge from the neonatal unit (discharge home or transfer to other neonatal unit), and demographic and clinical information were extracted in addition to reviewing computerised laboratory reporting systems as follows: gestational age, birth weight, sex, ethnicity, multiple pregnancy, days on respiratory support, days in supplementary oxygen, duration of stay, and post-menstrual age at discharge. Haematological data were extracted as follows: $\mathrm{Hb}, \%$ $\mathrm{HbF}, \% \mathrm{HbA}, \mathrm{HbF}: \mathrm{HbA}$ ratio, and blood transfusions (date, time, and volume as $\mathrm{ml} / \mathrm{kg}$ ). Episodes of infection were identified as definite (blood culture positive, CRP rise $>10$, and $\geq 5$ days antibiotics), probable (CRP rise $>10$ or positive blood culture, and $\geq 5$ days antibiotics), or not present. ROP screening data were recorded from patient notes for the duration of their ROP screening, according to established nomenclature. ${ }^{17}$ If there was no ROP at any time, the ROP outcome was recorded as stage 0 , while for any infant that did develop ROP, the worst stage in the worst eye was used as their outcome. The presence or absence of 'plus' (or 'pre-plus') disease was also noted. For those infants discharged to other neonatal units before retinopathy screening had been completed, data were extracted from BadgerNet, an electronic patient data management system.

\section{Data analysis}

Initial $\% \mathrm{HbF}$ (on admission after birth) and demographics on the cohort were extracted from the notes along with the results from their ROP screening examinations. Mean $\% \mathrm{HbF}$ (during inpatient stay) was derived using timeweighted averaging (multiplying each $\% \mathrm{HbF}$ value by the length of time between samples and then averaging the results for the whole period of an infant's inpatient stay). Initial univariate comparisons were carried out between those infants who developed any ROP vs those who did not, with respect to their $\mathrm{HbF}$ values. $\% \mathrm{HbF}$ from each full blood count was plotted against post-menstrual age. An ordered logistic regression model was used to calculate odds ratios (ORs) and 95\% confidence intervals (CIs) to estimate the association between the value of the $\% \mathrm{HbF}$ (either initial or mean of inpatient stay) with the most severe stage of ROP recorded for each baby $(0,1,2$, and 3). Corresponding adjusted ORs were derived after including gestation, birth weight, and total volume of red blood cell (RBC) transfusions in the logistic regression model.

Analysis was performed using STATA 10 (Stata Corp., College Station, TX, USA) and results are presented as number $(\%)$, mean (SD), median (interquartile range), or OR $(95 \% \mathrm{CI})$ as appropriate. Ethical approval was obtained from The NRES Committee North East Newcastle and North Tyne 2.

\section{Results}

A total of 42 infants were recruited between January 2012 and November 2013. No infants were excluded from the study. All parents approached for recruitment during the 
study agreed to be enrolled except the parents of one set of twins. Demographic data for the cohort, split by ROP status are displayed in Table 1.

A total of 37 neonates were inborn and five infants were outborn. All outborn infants were born in a local district general hospital, retrieved by the tertiary neonatal team and arrived in the tertiary neonatal unit before $10 \mathrm{~h}$ of age. Six infants died before ROP screening (three males and three females), of which four were inborn and two were outborn. Infants who developed ROP were more likely than infants who did not develop ROP to be from multiple births $(P=0.027)$, more preterm $(P<0.001)$, of lower birth weight $(P=0.007)$, to have had more transfusions $(P<0.001)$, larger volumes infused $(P<0.001)$, and to have spent longer on ventilators $(P<0.001)$ and continuous positive airway pressure $(P=0.028)$.

A total of 24 infants received a transfusion of red blood cells (RBCs) during their admission (57\%). Of the 36 infants who survived to ROP screening, all survived to discharge from the tertiary unit. Twenty-two of these infants did not develop ROP (52\% of the initial cohort). A total of 14 infants developed ROP (33\% of the initial cohort): stage $1(n=5)$, stage $2(n=4)$, stage $3(n=5,4$ of these infants also had plus disease, 4 of these infants received laser treatment, and 1 received intravitreal

Table 1 Demographics and ROP a outcome data

\begin{tabular}{lccr}
\hline Variable & No ROP & ROP & \multicolumn{1}{c}{ P } \\
\hline Male & $9(40.9 \%)$ & $5(35.7 \%)$ & 0.441 \\
Non-White ethnicity & $2(9.1 \%)$ & $0(0 \%)$ & 0.411 \\
Multiple birth & $11(50.0 \%)$ & $1(7.1 \%)$ & 0.027 \\
RBC $^{\mathrm{b}}$ transfusions & $0(0-1)$ & $3(1-5)$ & $<0.001$ \\
Total RBC transfusion $(\mathrm{ml} / \mathrm{kg})$ & $0(0-20)$ & $53(20-103)$ & $<0.001$ \\
Culture-positive sepsis & $2(9.1 \%)$ & $3(21.4 \%)$ & 0.297 \\
Gestation (weeks) & $29.2(1.1)$ & $26.6(1.6)$ & $<0.001$ \\
Birth weight $(\mathrm{g})$ & $1160(261)$ & $924(205)$ & 0.007 \\
Days on ventilator & $1(0-2)$ & $6(2-20)$ & $<0.001$ \\
Days on CPAP & $10(6-27)$ & $30(14-39)$ & 0.028 \\
Days on supplementary $\mathrm{O}_{2}$ & $28(7-51)$ & $50(21-73)$ & 0.051
\end{tabular}

Values are mean (SD), median (IQR), or $n(\%)$ as appropriate. ${ }^{\text {Retinopathy }}$ of prematurity. ${ }^{b}$ Red blood cell. bevacizumab), and grade $\geq 4(n=0)$. No infant developed retinal detachment (stage 4 or worse) during the study.

Those infants who did not develop ROP had higher initial haemoglobin levels (on admission) than infants who did develop ROP $(P=0.009)$, as shown in Table 2 and there was weak evidence that they have a lower initial $\% \mathrm{HbF}$ (83.3 vs $92.3 \%, P=0.06)$. Infants who developed ROP had significantly lower $(P=0.0001)$ mean $\% \mathrm{HbF}$ during their admission compared to those infants who did not develop ROP (Table 2).

When plotting $\mathrm{HbF} \%$ from each full blood count against post-menstrual age, there appears to be two populations. Those infants who developed ROP have noticeably lower $\mathrm{HbF} \%$ when compared to those infants without ROP (Figure 1).

The ordinal regression model produced similar results to the univariate associations above (Table 3). There was only weak evidence that initial $\mathrm{HbF} \%$ was associated with increasing risk of ROP grade $(P=0.070)$ and this association disappeared after adjusting for birth weight, gestation at birth, and volume of transfusion $(P=0.261)$. In contrast, there was strong evidence for an association between mean $\% \mathrm{HbF}$ and increasing risk of ROP grade in both the unadjusted $(P<0.001)$ and the adjusted analyses $(P=0.034)$.

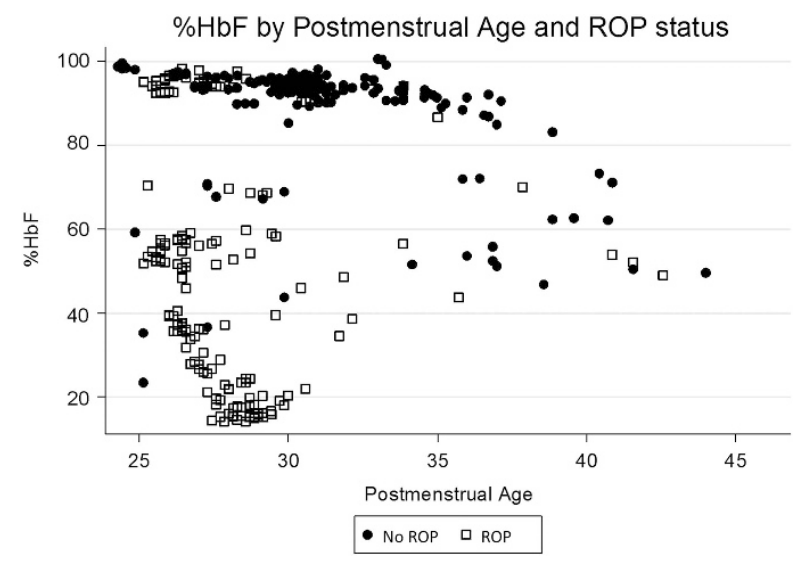

Figure 1 Percentage of $\mathrm{HbF}$ plotted against post-menstrual age (in weeks) showing ROP status.

Table 2 Comparison of haematological values between those infants who developed ROP and those that did not

\begin{tabular}{lccc}
\hline & No ROP $(95 \%$ CI $)(\mathrm{n}=22)$ & ROP $(95 \%$ CI $)(\mathrm{n}=14)$ & P-value \\
\hline Initial $\mathrm{Hb}(\mathrm{g} / \mathrm{l})$ & $162.5(153.2,171.8)$ & $143.6(133.3,153.9)$ & 0.009 \\
Mean $\mathrm{Hb}(\mathrm{g} / \mathrm{l})$ & $134.9(116.0,153.7)$ & $112.4(102.8,122.1)$ & 0.06 \\
Initial \%HbF & $92.3(89.9,94.7)$ & $83.3(71.1,95.5)$ & 0.06 \\
Mean \%HbF & $91.87(87.2,96.5)$ & $61.75(44.5,79.0)$ & 0.0001 \\
\hline
\end{tabular}


Table 3 Association between haematological values and ROP

\begin{tabular}{lcc}
\hline Variable & OR $(95 \%$ CI $)$ & Adjusted $^{\text {a }}$ OR $(95 \%$ CI $)$ \\
\hline Initial HbF\% & $0.96(0.93-1.00)$ & $0.97(0.91-1.03)$ \\
Mean $\mathrm{HbF} \%$ & $0.94(0.90-0.97)$ & $0.94(0.90-0.99)$ \\
\hline
\end{tabular}

Abbreviations: $\mathrm{CI}$, confidence interval; OR, odds ratio. ${ }^{\text {a }}$ Adjusted for birth weight, gestation, and total transfusion volume.

\section{Discussion}

While very preterm infants who developed ROP had similar initial $\% \mathrm{HbF}$ when compared to those who did not develop ROP, we observed that as hypothesised, mean $\% \mathrm{HbF}$ was significantly lower in the ROP group during their inpatient stay than in the babies who did not develop ROP. While initial $\mathrm{HbF}$ was only weakly associated with ROP, it is not surprising that the mean $\mathrm{HbF}$ showed a strong association as this represents the total period of exposure to high or low $\mathrm{HbF}$. A lower $\% \mathrm{HbF}$ may be a proxy or surrogate marker for sickness, as neonates who are more unwell will be more likely to require and receive blood transfusions. We therefore included volume of blood transfused as an approximate proxy marker of illness severity in the logistic regression model, and an association still seemed to hold between lower $\% \mathrm{HbF}$ and ROP development. There is physiological evidence supporting left shift of the oxyhaemoglobin dissociation curve with increasing $\% \mathrm{HbF}$. It is therefore biologically plausible that a lower $\mathrm{HbF}$ concentration (and higher $\mathrm{HbA}$ concentration) provides greater oxygen delivery to the developing retina. Evidence of this effect (ROP) has been found in our study. It may therefore be that maintaining higher $\mathrm{HbF}$ levels for longer confers some protection against ROP development.

We recognise limitations with our study, notably a small sample size. Identifying causal factors in ROP development is not possible in this small cohort study due to the large number of variables and confounding factors over this time period, as well as a relatively heterogeneous patient population. We considered supplemental oxygen therapy to be part of the causal pathway leading to ROP development, rather than as a confounder, and did not therefore adjust for it in these models. In a larger study, it would be useful to explore in more detail the relationship between supplemental oxygen, intercurrent illness, and blood transfusions on $\% \mathrm{HbF}$ and on the development of ROP. There is a need for further research to establish evidence for a potential causal relationship between $\mathrm{HbF} \%$ and ROP risk.

Various interventions including laser therapy and angiogenesis inhibitors are utilised to manage established $\mathrm{ROP}$ and much research is ongoing in this area. However, if high-risk infants can be recognised and there is a possibility of enhancing intrinsic protective factors, then
ROP development could potentially be minimised or prevented. Delayed cord clamping has been shown to deliver additional blood to the newborn from the placental bed. ${ }^{18,19}$ This could potentially reduce or delay the need for subsequent transfusion, in turn facilitating maintenance of $\mathrm{HbF}$. There is already reluctance to transfuse liberally in preterm neonates due to NEC risk, exposure to donors, and risk of transfusion reaction, but risk of ROP development might also need to be considered if further research supports the hypothesis that early loss of $\mathrm{HbF}$ predisposes an infant to developing ROP.

\section{Conclusions}

To our knowledge, this is the first study to investigate, and find an association between $\mathrm{HbF}$ concentration, blood transfusion, and ROP development. It is possible that $\mathrm{HbF}$ is a protective factor against ROP, and that transfusion of adult $(\mathrm{HbA})$ blood may play a part in ROP development by suddenly making more oxygen available to the developing retina and downregulating VEGF, resulting in arrest of the advancing front of retinal vasculature. Subsequent reduction of oxygen supply leads to ischaemia of the unvascularised peripheral retina and marked upregulation of VEGF with the development of fundoscopic signs of ROP. Larger studies are required to investigate these associations further.

\section{Summary}

What was known before

- Retinopathy of prematurity (ROP) is a major cause of morbidity in the preterm population.

- Oxygen is known to be the predominant causal factor in ROP development.

- ROP has been shown to be associated with blood transfusion.

What this study adds

- Blood transfusion markedly reduces the foetal haemoglobin $(\mathrm{HbF})$ concentration.

- Those infants who develop ROP have significantly lower mean $\mathrm{HbF}$ levels.

- Maintaining higher $\mathrm{HbF}$ levels may be protective against ROP.

\section{Conflict of interest}

The authors declare no conflict of interest.

\section{Author contributions}

CS: contributed to study design, recruited patients, designed data extraction proforma, extracted all case 
notes and laboratory data, assisted with data analysis, and drafted the scientific paper. AJ: contributed to study design, recruited patients, assisted with data analysis, and edited the scientific paper. DO: contributed to study design, recruited patients, conducted statistical analysis, and edited the scientific paper. CW: contributed to study design, performed all ROP screening, assisted with data analysis, and edited the scientific paper. RM: Principal Investigator, study inception and design, secured charitable funding, and edited the scientific paper.

\section{Acknowledgements}

Sharon Evans, Lead Biomedical Scientist for Special Haematology, Blood Sciences, North Bristol Trust Southmead Hospital—analysis of all blood samples using HPLC. Kirsty Stevenson, Laboratory technician, The Unit for the Support of Trials and Research, Dpt. of Clinical Biochemistry, Bristol Royal Infirmary-identification of enrolled infant blood samples and forwarding to analysis laboratory. This study was funded by David Telling Charitable Trust grant.

\section{References}

1 Campbell K. Intensive oxygen therapy as a possible cause of retrolental fibroplasias; a clinical approach. Med J Aust 1951; 2(2): 48-50.

2 Askie LM, Brocklehurst P, Darlow BA, Finer N, Schmidt B, Tarnow-Mordi W et al. NeOProM Collaborative Group. NeOProM: neonatal oxygenation prospective meta-analysis collaboration study protocol. BMC Pediatr 2011; 11: 6.

3 Stenson BJ, Tarnow-Mordi WO, Darlow BA et al. BOOST II United Kingdom Collaborative Group; BOOST II Australia Collaborative Group; BOOST II New Zealand Collaborative Group. Oxygen saturation and outcomes in preterm infants. N Engl J Med 2013; 368 (22):2094-2104.

4 Carlo WA, Finer NN, Walsh MC, Rich W, Gantz MG et al. SUPPORT Study Group of the Eunice Kennedy Shriver NICHD Neonatal Research Network Target ranges of oxygen saturation in extremely preterm infants. N Engl J Med 2010; 362 (21):1959-1969.

5 Fortes Filho JB, Eckert GU, Procianoy L, Barros CK, Procianoy RS. Incidence and risk factors for retinopathy of prematurity in very low and extremely low birth weight infants in a unit-based approach in Southern Brazil. Eye (Lond) 2009; 23(1): 25-30.
6 Dani C, Reali MF, Bertini G, Martelli E, Pezzati M, Rubaltelli FF. The role of blood transfusions and iron intake on retinopathy of prematurity. Early Hum Dev 2001; 62(1): $57-63$.

7 Hellstrom A, Hard AL, Engstrom E, Niklasson A, Andersson E, Smith L et al. Early weight gain predicts retinopathy in preterm infants: new, simple, efficient approach to screening. Pediatrics 2009; 123(4): e638-e645.

8 Hesse L, Eberl W, Schlud M, Poets CF. Blood transfusion, iron load and retinopathy of prematurity. Eur J Pediatr 1997; 156(6): 465-470.

9 Cooke RW, Clark D, Hickey-Dwyer M, Weindling AM. The apparent role of blood transfusions in the development of retinopathy of prematurity. Eur J Pediatr 1993; 152(10): 833-836.

10 Englert JA, Saunders RA, Purohit D, Hulsey TC, Ebeling M. The effect of anaemia on retinopathy of prematurity in extremely low birth weight infants. J Perinatol 2001; 21(1): $21-26$.

11 Terrenato L, Bertilaccio C, Spinelli P, Colombo B. The switch from haemoglobin $\mathrm{F}$ to $\mathrm{A}$ : the time course of qualitative and quantitative variations of haemoglobin after birth. $\mathrm{Br} J$ Haematol 1981; 47(1): 31-41.

12 Bard H. The postnatal decline of hemoglobin F synthesis in normal full term infants. J Clin Invest 1975; 55(2): 395-398.

13 Guyton AC. Regulation of cardiac output. Anesthesiology 1968; 29: 314-326.

14 Oski FA. The erythrocyte and its disorders. In: Nathan A, Oski FA (eds). Haematology of Infancy and Childhood. WB Saunders: Philadelphia, PA, USA, 1993, pp 18-43.

15 De Halleux V, Truttmann A, Gagnon, Bard H. The effect of blood transfusion on the hemoglobin oxygen dissociation curve of very early preterm infants during the first week of life. Semin Perinatol 2002; 26(6): 411-415.

16 UK Retinopathy of Prematurity Guideline, 2008. Available at: https://www.rcophth.ac.uk/wp-content/ uploads /2014/12/2008-SCI-021-Guidelines-Retinopathy-ofPrematurity.pdf (accessed on January 2016).

17 International Committee for the Classification of Retinopathy of Prematurity. The International Classification of Retinopathy of Prematurity revisited. Arch Ophthalmol 2005; 123(7): 991-999.

18 Elimian A, Goodman J, Escobedo M, Nightingale L, Knudtson E, Williams M. Immediate compared with delayed cord clamping in the preterm neonate: a randomised controlled trial. Obstet Gynecol 2014; 124(6): 1075-1079.

19 McDonald SJ, Middleton P, Dowswell T, Morris PS. Cochrane in context: effect of timing of umbilical cord clamping in term infants on maternal and neonatal outcomes. Evid Based Child Health 2014; 9(2): 398-400. 